\title{
P.269 THE AMPAKINE CX717 HAS A RAPID, BUT SHORT-LASTING ANTIDEPRESSANT-LIKE ACTIVITY IN THE RAT
}

Marta Gordillo-Salas ${ }^{1}$, Jun Ren ${ }^{2}$, John Greer ${ }^{2}$ \& Albert Adell,

${ }^{1}$ Institute of Biomedicine \& Biotechnology of Cantabria, IBBTEC (CSIC-UC), Santander, Spain.

2 University of Alberta, Department of Physiology, Edmonton, Canada.

${ }^{3}$ CIBERSAM, Biomedical Research Networking Center Consortium, Mental Health, Santander, Spain.

\section{INTRODUCTION}

Major depressive disorder (MDD) is the most prevalent of psychiatric disorders. Currently available antidepressant drugs increase the availability of certain neurotransmitters in the brain. Briefly, it has been proposed that increasing the activity of take up to seral weeks to be elfeclive. In rech years, $n$ ew approaches have ent Interestingly, these novel therapies require the stimulation of AMPA glutamate receptors to exert their antidepressant effects. Consequently, it has been proposed that ampakines may be associated with mood enhancing properties.

\section{AIM OF THE STUDY}

The aim of the present work was to investigate the antidepressant-like effects of the ampakine $\mathrm{CX717}$ in the forced swim The aim of the present work was to investigate the antidepressant-like effects of the ampakine CX717 in the forced swim in intracellular signaling pathways and cortical transmitter release over time were also examined.

\section{METHODS}

Animals: Male Sprague-Dawley rats (Envigo) weighing 280-350g.

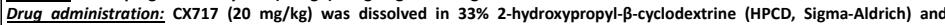
injected IP. This solution was further diluted to $300 \mu \mathrm{M}$ in artificial CSF for intracortical infusion through dialysis probes implanted bilaterally in the medial prefrontal cortex (MPFC; AP: $+3.2, \mathrm{ML}: \pm 0.5$ and DV: -5.4 .

In vivo microdialysis: Extracellular levels of serotonin (5-HT), noradrenaline (NA), dopamine (DA) and glutamate (Glu) were determined using an Alexys ${ }^{\circledR}$ Analyzer (Antec, The Hetherlands).

Western blotting: After the administration of $20 \mathrm{mg} / \mathrm{kg}$ of CX717, animals were killed by decapitation, their brains removed from the skulls, and mPFCs dissected out on ice and rapidly stored at $-80^{\circ} \mathrm{C}$. The sources and dilution of primary antibodies

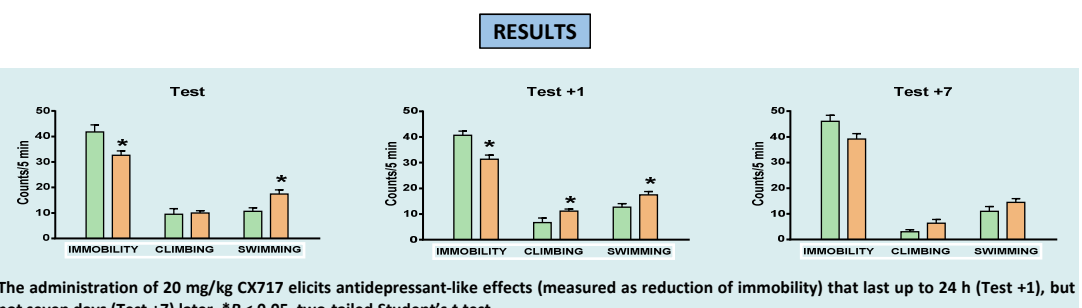

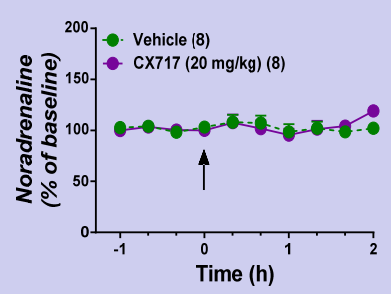
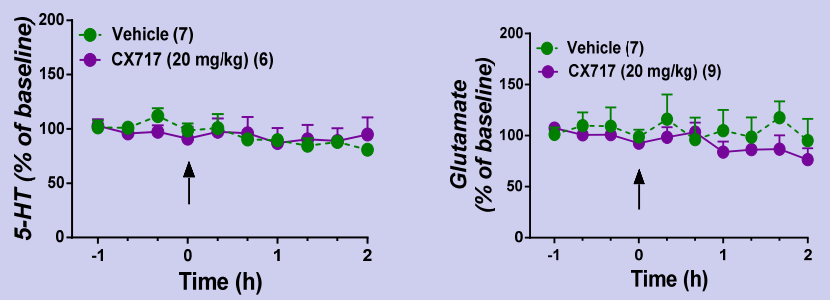

The administration of $20 \mathrm{mg} / \mathrm{kg}$ CX717 does not alter extracellular concentrations of monoamines and glutamate. BDNF

p11

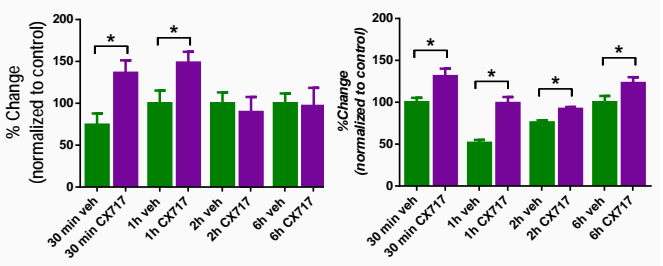

The intraperitoneal administration of $20 \mathrm{mg} / \mathrm{kg}$ CX717 elicits rapid increases of BDNF and $\mathrm{p} 11$ in the mPFC. ${ }^{*} P<0.05$, two-tailed Student's $t$-test.
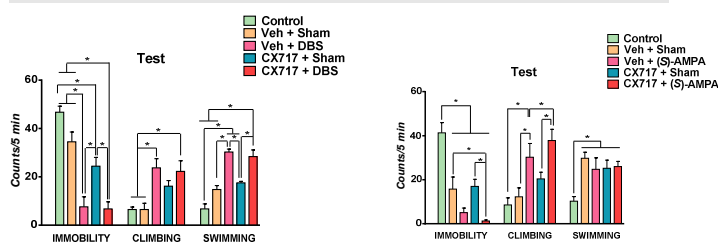

Test +1

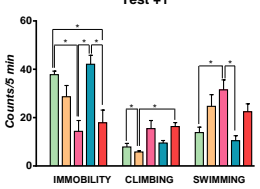

Test +
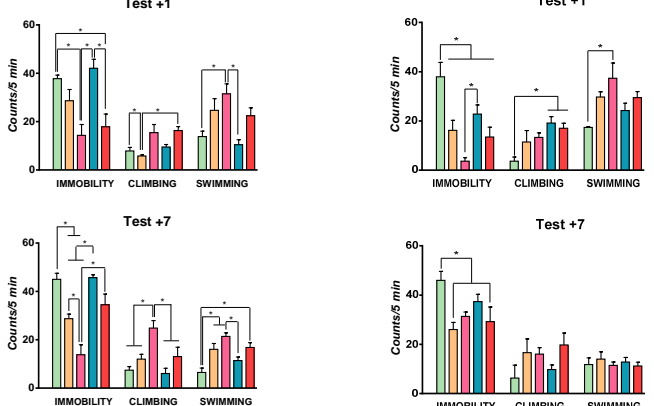

Test +7

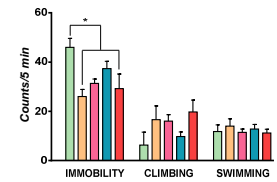
in the $\mathrm{MPFC}$.

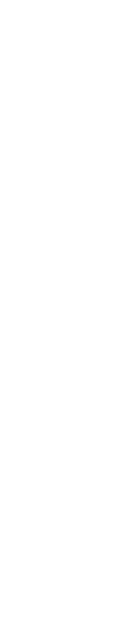

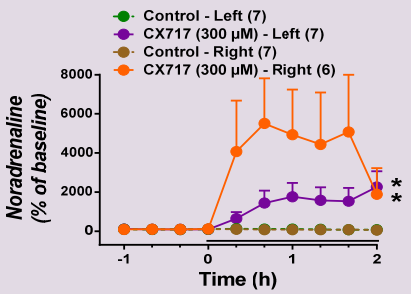
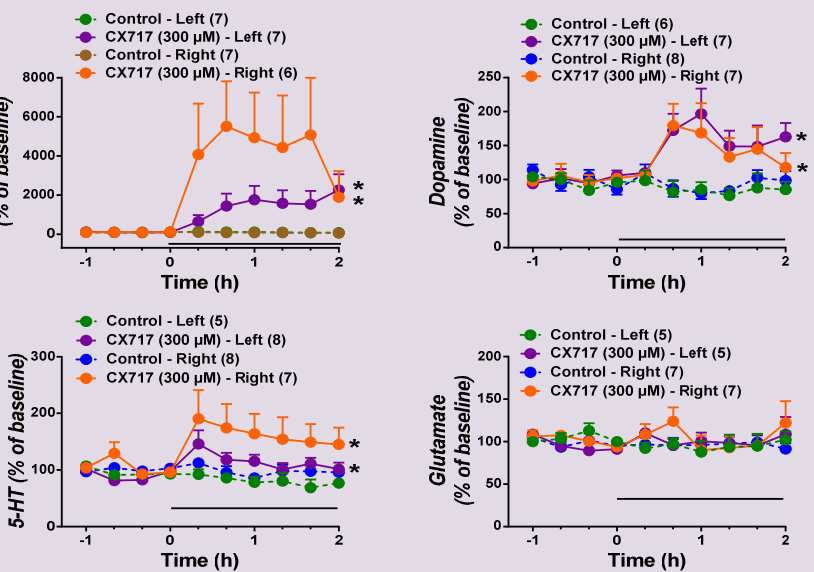

The local infusion of $300 \mu \mathrm{M} C X 717$ bilaterally in the mPFC (black bar) increases the extracellular concentration of monomines without altering that of glutamate. ${ }^{*} P<0.05$, Newman-Keul's test after significant repeated measures ANOVA.

GluA1

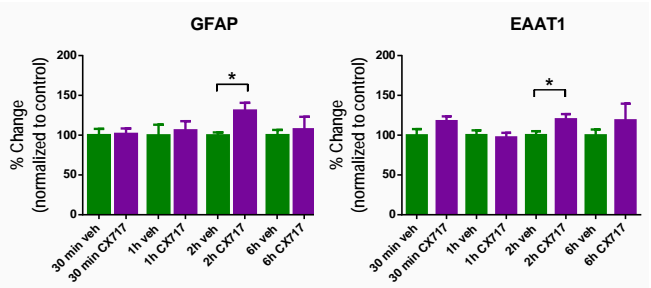

The IP administration of $20 \mathrm{mg} / \mathrm{kg}$ CX717 does not change the protein level of GluA1

The intraperitoneal administration of $20 \mathrm{mg} / \mathrm{kg}$ CX717 elicits more delayed increase of the glia markers GFAP and EAAT1 in the MPFC. ${ }^{*} P<0.05$, two-tailed Student's $t$-test.

\begin{abstract}
CONCLUSIONS
CX717 has a rapid, but short-lasting, antidepressant-like activity.

CX717 does not potentiate the antidepressant response of DBS or intra-cortical infusion of (S)-AMPA, most likely because the effects of such treatments are already maximal.

The increase in prefrontal monoamines caused by CX717 is only observed after local infusion of the drug in the mPFC. It is possible that a sustained administration of the drug is needed to alter prefrontal monoamine release.

The rapid antidepressant-like effects of $\mathrm{CX717}$ are likely caused by a fast release of BDNF and synthesis of p11.

A delayed increase in glial markers (GFAP and EAAT1) might also contribute to these effects.
\end{abstract}

